\title{
Work-in-Progress: Automatic Generation of Application-Specific FPGA Overlays
}

\author{
Danielle Tchuinkou Kwadjo \\ University of Florida \\ dtchuinkoukwadjo@ufl.edu
}

\author{
Joel Mandebi Mbongue \\ University of Florida \\ jmandebimbongue@ufl.edu
}

\author{
Christophe Bobda \\ University of Florida \\ cbobda@ece.ufl.edu
}

\begin{abstract}
This work proposes a generic flow for designing application-specific FPGA overlays that can achieve bare metal performance while improving productivity, resulting in increased adoption of FPGAs by software developers. The proposed approach relies on automatic extraction of kernels in high-level language applications. Extracted Kernels are then systematically translated into optimized hardware circuits using RapidWright, which allows bypassing HDL design flows. Initial results show up to $19 \times$ productivity improvement over regular overlays, and higher Fmax compared to bare metal in several cases.
\end{abstract}

\section{KEYWORDS}

FPGA, Overlay, LLVM, RapidWright

\section{ACM Reference Format:}

Danielle Tchuinkou Kwadjo, Joel Mandebi Mbongue, and Christophe Bobda. 2019. Work-in-Progress: Automatic Generation of Application-Specific FPGA Overlays. In 2019 International Conference on Compliers, Architectures and Synthesis for Embedded Systems (CASES'19), October 13-18, 2019, New York, NY, USA. ACM, New York, NY, USA, 2 pages. https://doi.org/10.1145/3349569. 3351533

\section{INTRODUCTION}

FPGA overlays have been developed to increase the programmability and adoption of FPGAs while achieving high-performance. They generally use coarse-grained processors in a communication infrastructure allowing parallel processing and data exchange. Unfortunately, the programmability gains of FPGA overlays are more than offset by massive performance loss, and increased power and resource consumption, mainly because they most often embed more features than what is actually needed to run applications. Current FPGA overlays mostly present architectures tailored for specific applications that relies on homogeneous processing units not adaptable to different use cases $[1,6]$. Further, some research discuss the automatic generation of hardware accelerators. But, they either require expertise on domain-specific languages, imposing a learning burden on developers [5], or they necessitate specific types of inputs, not reflecting how developers would typically implement applications [2], all that without necessarily achieving high performance. Tools such as Vivavo HLS allow to write high-level codes

Permission to make digital or hard copies of all or part of this work for personal or classroom use is granted without fee provided that copies are not made or distributed for profit or commercial advantage and that copies bear this notice and the full citation on the first page. Copyrights for components of this work owned by others than ACM must be honored. Abstracting with credit is permitted. To copy otherwise, or republish, to post on servers or to redistribute to lists, requires prior specific permission and/or a fee. Request permissions from permissions@acm.org.

CASES'19, October 13-18, 2019, New York, NY, USA

(C) 2019 Association for Computing Machinery.

ACM ISBN 978-1-4503-6925-1/19/10 ..\$15.00

https://doi.org/10.1145/3349569.3351533 for FPGAs, but still necessitate hardware skills and long compilation time. In this paper, we propose an application-specific FPGA overlay (AS-Overlay) design flow for productivity and performance improvement. At first, the flow leverages Level Virtual Machine (LLVM) [3], a compilation and code instrumentation framework, for automatic extraction of kernels within applications. Then, hardware is systematically produced with no HDL generation using RapidWright [4], an open source framework for custom netlist manipulation from Xilinx Research Labs.

\section{PROPOSED APPROACHED}

\subsection{Design Flow}

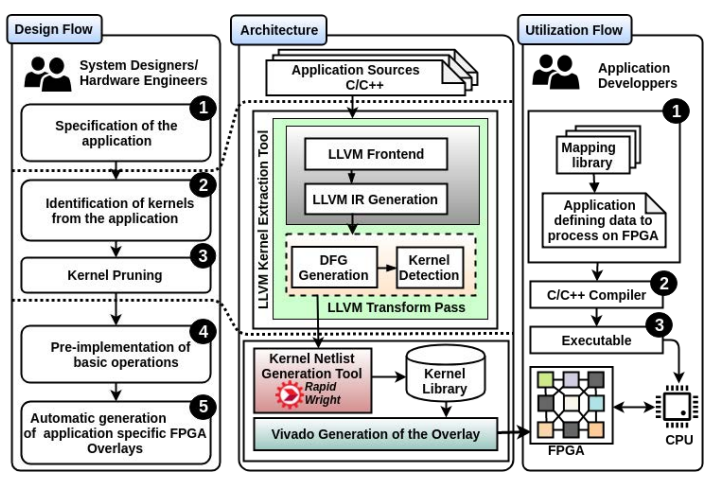

Figure 1: Design Flow, Utilization Flow and Architecture

We aim to build FPGA overlays that can compete with bare metal implementations. Figure 1 summarizes the design flow, the architecture supporting the application-specific overlay generation, and the utilization flow. The design flow starts by inspecting the intermediate representation (IR) of applications at compile time with LLVM to extract compute-intensive sections that we call kernels. Next, we manually pre-synthesize basic operations from the IR using vendor tools: this step is done exactly once, and the synthesized netlists are reusable in several applications. Finally, we use RapidWright to combine the pre-synthesized basic operations into hardware kernels. Hardware kernels thus generated are embedded within processing units of an arbitrary overlay architecture in place of general-purpose ALUs, and Vivado is used for placement and routing. Users only call high-level functions described in a custom API with the data to process, removing the need for hardware expertise.

\subsection{Hardware Generation}

An LLVM transform pass is implemented to extract kernel from applications based on the gspan algorithm [7]. Code sections are identified as kernels if their frequency of appearance is greater than a threshold. Kernels are then pruned to select only operations that can actually be mapped on FPGAs: applications are written 
for Von Newman CPUs, which incurs the insertion of load/store, and casting instructions that are not actually needed on FPGAs.

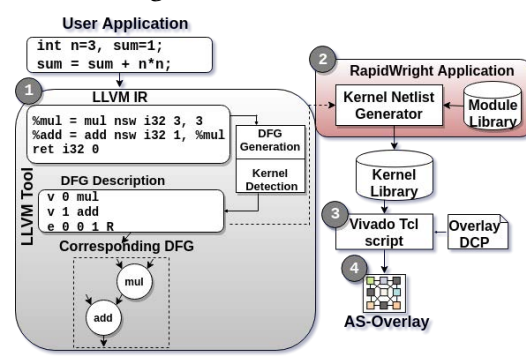

Figure 2: Generation Example Initially, the function implemented by a PE in the overlay layout is defined as a black-box. We leverage the pre-implemented design flow of RapidWright [4] to produce netlists from kernels previously identified with LLVM. The first step consists in synthesizing basic operations from LLVM IR out-of-context (OOC) with Vivado to create a library of Modules. This stage implies a manual implementation (through HDL or HLS) of operations to combine into kernels by an engineer, with the advantage that this step is done once. In the following stage, an application built on top of RapidWright assembles pre-implemented modules according to the kernel descriptions. The hardware kernels thus generated are returned as design checkpoint files (DCP) and define the functions executed by PEs. A custom Vivado Tcl script reads-in kernel DCPs into PE black-boxes. Finally, placement and routing are run with Vivado. Figure 2 summarizes the steps for the automatic generation of an FPGA overlay.

\section{EXPERIMENTAL RESULTS}

Designs are implemented on a Xilinx Kintex UltraScale+ FPGA (xcku5p-ffvd900-2-i). Hardware generation is conducted with Vivado HLx Editions v2018.2, and RapidWright v2018.2.5-beta allows assembling hardware kernels. We ran Vivado, RapidWright, and the LLVM kernel mining on a computer with an Intel Corei3-8130U CPU@2.20GHz×4 and 8Gb of RAM. We compare manual implementation (Bare Metal), a regular overlay with an ALU in each PE, and AS-Overlays for each application, with $3 \times 3$ PEs. For Fmax and productivity studies summarized in Table 1 , we introduced a Phase-Locked Loop generating a 300MHz clock on each testing architecture. The idea was to observe the maximum frequency and how long would the compilation take. AS-Overlays achieved up to $1.47 \times$ improved Fmax compared to regular overlays. This is caused by the muxes in general-purpose ALUs, introducing substantial delays on datapaths. On the other hand, bare metal implementations achieved higher Fmax compared to AS-Overlays on outer product and Robert cross filter. It is because vendor tools such as Vivado often produce high performance results for small modules [4]. In this case of figure, outer product and Robert cross are respectively a set of independent multiplications and subtractions followed by comparisons, simple enough give to bare metal a $1.09 \times$ Fmax advantage over AS-Overlays. That advantage is nevertheless lost on more complex functions such as image smoothing, highlighting the benefits of using the RapidWright pre-implemented flow as smaller modules can be pre-implemented to achieve maximum frequency, and later be assembled with minimal QoR loss. Reported compilation times show that Kernel netlist generation with RapidWright and loading with Vivado read_checkpoint command, outperforms by about $5 \times$ Vivado synthesis both in Regular overlays and bare metal implementations. Table 1 finally demonstrates that the proposed
AS-overlay generation flow can provide up to $19 \times$ productivity improvement over regular overlays on tested benchmarks.

Ma et al. [5] reported a productivity improvement between $170 \times$ and $214 \times$, which only accounts synthesis. Further, they used benchmarks and FPGA different from ours. Similar observations can be made on other related work, making it challenging to establish fair comparisons.

Table 1: Productivity Analysis \& Maximum Frequency

\begin{tabular}{|c|c|c|c|c|c|}
\hline \multirow{2}{*}{\multicolumn{2}{|c|}{$\begin{array}{c}3 \times 3 \text { Overlay } \\
\text { Generation Flow }\end{array}$}} & \multicolumn{4}{|c|}{ Applications } \\
\hline & & $\begin{array}{c}\text { Matrix } \\
\text { Mult }\end{array}$ & $\begin{array}{c}\text { Outer } \\
\text { Product }\end{array}$ & $\begin{array}{c}\text { Robert } \\
\text { Cross }\end{array}$ & Smoothing \\
\hline \multirow{6}{*}{ 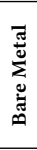 } & Synthesis & 26 & 17 & 35 & 35 \\
\hline & Optimization & 5 & 2 & 6 & 34 \\
\hline & Placement & 28 & 23 & 44 & 85 \\
\hline & Routing & 68 & 59 & 55 & 1064 \\
\hline & Total(Seconds) & 127 & 101 & 140 & 1218 \\
\hline & $\operatorname{Fmax}(\mathrm{MHz})$ & 365 & 488 & 348 & 231 \\
\hline \multirow{2}{*}{ 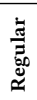 } & Vivado Flow $\rightarrow$ & $\begin{array}{c}\text { Synth. } \\
40\end{array}$ & $\begin{array}{c}\text { Opt. } \\
27\end{array}$ & $\begin{array}{c}\text { Place. } \\
111\end{array}$ & $\begin{array}{c}\text { Routing } \\
1457\end{array}$ \\
\hline & $\begin{array}{l}\text { Total (Seconds) } \\
\text { Fmax }(\mathrm{MHz})\end{array}$ & \multicolumn{4}{|c|}{$\begin{array}{c}1635 \text { (27 minutes } 15 \text { seconds) } \\
304\end{array}$} \\
\hline \multirow{7}{*}{ 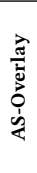 } & Kernel Gen. & 3.89 & 3.48 & 3.55 & 4.34 \\
\hline & Kernel Load. & 8.12 & 5.83 & 4.12 & 7.8 \\
\hline & Optimization & 5 & 4 & 3 & 33 \\
\hline & Placement & 46 & 19 & 24 & 83 \\
\hline & Routing & 65 & 54 & 117 & 646 \\
\hline & Total (Seconds) & 128.01 & 86.31 & 154.67 & 774.14 \\
\hline & Fmax $(\mathrm{MHz})$ & 435 & 447 & 318 & 308 \\
\hline
\end{tabular}

\section{CONCLUSION}

We presented an approach for automatic generation of ApplicationSpecific FPGA Overlays capable of providing bare metal performances. The approach extracts kernel from applications, and automatically builds a tailored accelerator. Experimental evaluations demonstrated the viability of our approach with significant productivity and Fmax improvement over regular FPGA overlays. Future works will investigate the possibility of generating hardware capable of reaching higher Fmax. We also intend to study how the power consumption scales in AS-Overlays compared to bare metal implementations and regular overlays.

\section{ACKNOWLEDGEMENT}

This work was supported by the ONR under the Grant CCN 040217643-21-0000.

\section{REFERENCES}

[1] Alexander Brant and Guy GF Lemieux. 2012. ZUMA: An open FPGA overlay architecture. In Field-Programmable Custom Computing Machines (FCCM), 2012 IEEE 20th Annual International Symposium on. IEEE, 93-96.

[2] David Koeplinger, Raghu Prabhakar, Yaqi Zhang, Christina Delimitrou, Christos Kozyrakis, and Kunle Olukotun. 2016. Automatic generation of efficient accelerators for reconfigurable hardware. In 2016 ACM/IEEE 43rd Annual International Symposium on Computer Architecture (ISCA). Ieee, 115-127.

[3] Chris Lattner and Vikram Adve. 2004. LLVM: A compilation framework for lifelong program analysis \& transformation. In Proceedings of the international symposium on Code generation and optimization: feedback-directed and runtime optimization. IEEE Computer Society, 75.

[4] Chris Lavin and Alireza Kaviani. 2018. Rapidwright: Enabling custom crafted implementations for fpgas. In 2018 IEEE 26th Annual International Symposium on Field-Programmable Custom Computing Machines (FCCM). IEEE, 133-140.

[5] Sen Ma, Zeyad Aklah, and David Andrews. 2015. A run time interpretation approach for creating custom accelerators. In Field Programmable Logic and Applications (FPL), 2015 25th International Conference on. IEEE, 1-4.

[6] Joel Mandebi Mbongue, Danielle Tchuinkou Kwadjo, and Christophe Bobda. 2018. FLexiTASK: A Flexible FPGA Overlay for Efficient Multitasking. In Proceedings of the 2018 on Great Lakes Symposium on VLSI. ACM, 483-486.

[7] Xifeng Yan and Jiawei Han. 2002. gspan: Graph-based substructure pattern mining. In Data Mining, 2002. ICDM 2003. Proceedings. 2002 IEEE International Conference on. IEEE, 721-724. 